\title{
Efecto del consumo de café regular y café descafeinado sobre la glicemia en adultos jóvenes
}

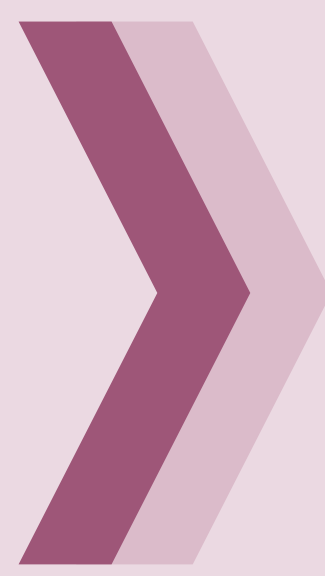

\author{
Effect of regular and \\ decaffeinated coffee \\ consumption on glycemia in \\ young adults
}

\section{Juan Jorge Huamán-Saavedra, ${ }^{1}$ Alberto Manuel Herrera-Aquino, ${ }^{2,3}$ Carito Yeniffer Nery-Zavaleta, ${ }^{2,4}$ Rocío del Pilar Zamora-Chávez, ${ }^{24}$ Julio Hilario-Vargas ${ }^{5}$}

Facultad de Medicina de la Universidad Nacional de Trujillo (UNT), Trujillo, Perú.

1. Doctor en Medicina. Patólogo clínico. Profesor principal del departamento de Ciencias Básicas-Medicina de la UNT, Trujillo, Perú.

2. Miembro de la Sociedad Científica de Estudiantes de Medicina de la UNT.

3. Estudiante del quinto año de Medicina Humana de la UNT.

4. Estudiante del cuarto año de Medicina Humana de la UNT.

5. Doctor en Ciencias Biomédicas. Profesor asociado del departamento de Fisiología, UNT.

\section{RESUMEN}

Oвjetivo. Analizar el efecto del consumo de café regulary café descafeinado sobre la glicemia en adultos jóvenes. Material y métodos. Diseño experimental. Se seleccionaron 42 estudiantes de medicina, divididos en tres grupos: G1 consumió café regular; G2, café descafeinado; G3, un placebo. Se realizó la prueba de tolerancia oral a glucosa (PTOG) antes del consumo, a las 4 y 8 semanas. Los datos fueron evaluados mediante la prueba $t$ de Student para muestras relacionadas pre y posprueba de cada grupo, método estadístico ANOVA y prueba de Tukey; con significancia $p<0,05$. Resultados. Antes del consumo, la glicemia en $\mathrm{G} 1, \mathrm{G} 2$ y $\mathrm{G} 3$ a los 0 minutos de la PTOG fue de $91,07 \mathrm{mg} / \mathrm{dL}, 91,86 \mathrm{mg} / \mathrm{dL}$ y $94,14 \mathrm{mg} /$ $\mathrm{dL}$, respectivamente, y a los 120 minutos de $114,07 \mathrm{mg} /$ $\mathrm{dL}, 116,50 \mathrm{mg} / \mathrm{dL}$ y $110,57 \mathrm{mg} / \mathrm{dL}$, respectivamente. A las 8 semanas, a los 0 minutos de la PTOG: G1 tuvo una glicemia de $87,64 \mathrm{mg} / \mathrm{dL}, \mathrm{G} 2$ de $95,57 \mathrm{mg} / \mathrm{dL}$ y G3 de $95,57 \mathrm{mg} / \mathrm{dL}$ sin encontrar cambios significativos, a los 120 minutos: G1 una glicemia de $98 \mathrm{mg} / \mathrm{dL}$ con una disminución significativa de 14,09\%, G2 de 123,29 mg/ dL y $\mathrm{G} 3$ de $113,21 \mathrm{mg} / \mathrm{dL}$, sin cambios significativos. Conclusión. El consumo de café regular disminuye significativamente la glicemia a los 120 minutos de la PTOG en adultos jóvenes.

Palabras clave: café regular; café descafeinado; glicemia

\begin{abstract}
Goal. Evaluate the effect of consumption of regular coffee and decaffeinated coffee on glycemia in young adults. Material AND Methods. Experimental design. Were selected 42 students from Medical School, divided into three groups; which consumed; G1: regular coffee, G2: decaffeinated coffee, G3: a placebo. The glycemia was measured, by the oral glucose tolerance test (OGTT), to the beginning, at 4 weeks and 8 weeks. The data were evaluated by t of student's test for related samples before and after test in each group. Statistics: ANOVA and Tukey test; with significance $p<0,05$. REsults. Before the test with coffee, the glucose level in $\mathrm{G} 1, \mathrm{G} 2$ and $\mathrm{G} 3$ at 0 minutes of OGTT was $91,07,91,86$ and $94,14 \mathrm{mg} / \mathrm{dL}$, respectively, and $114,07 \mathrm{mg} / \mathrm{dL}, 116,50$ and $110,57 \mathrm{mg} / \mathrm{dL}$ at 120 minutes, respectively. At 8 weeks, at 0 minutes of OGTT, $\mathrm{G} 1 \mathrm{had} 87,64 \mathrm{mg} / \mathrm{dL} ; \mathrm{G} 2,95,57 \mathrm{mg} / \mathrm{dL}$ and $\mathrm{G} 3,95,57 \mathrm{mg} / \mathrm{dL}$ glycemia without significant changes; and at 120 minutes: G1 had $98 \mathrm{mg} / \mathrm{dL}$ with a significant decrease (14,09\%); G2, $123,29 \mathrm{mg} / \mathrm{dL}$ and $\mathrm{G} 3,113,21 \mathrm{mg} / \mathrm{dL}$ without significant changes. Conclusion. The consumption of regular coffee decrease glycemia significantly at 120 minutes of OGTT, in young adults.
\end{abstract}

KEYWORDs: regular coffee; decaffeinated coffee; glycemia. 


\section{INTRODUCCIÓN}

Café, bebida que se obtiene por infusión a partir de la planta Coffea arábica L, contiene aminoácidos, polisacáridos, azúcares, triglicéridos, ácidos, compuestos fenólicos (ácido clorogénico) y cafeína. ${ }^{1}$ En Trujillo, $70 \%$ de las personas consume aproximadamente $460 \mathrm{~g}$ de café instantáneo anualmente. ${ }^{2}$ Se puede encontrar dos tipos de café instantáneo, el café regular (café cafeinado) y el café descafeinado. ${ }^{1}$ El primero contiene $3142 \mathrm{mg}$ de cafeína en $100 \mathrm{~g}$ de café y el segundo, solo $122 \mathrm{mg}$. ${ }^{3}$ Un consumo inferior de $300 \mathrm{mg}$ de cafeína al día no supone ningún problema de salud. ${ }^{4}$

Existen diversos estudios en adultos que indican posibles efectos del café sobre la glicemia. En Estados Unidos; en 2010, se reportó que el consumo de café regular aumentaba la glicemia mientras que el café descafeinado la disminuía, en 2011, en personas con sobrepeso el café regular y café descafeinado no alteraron significativamente la glicemia. ${ }^{6}$ En Japón, en 2012, en varones con sobrepeso, el café regular disminuyó la glicemia y el café descafeinado no la alteró. En 2013, en Grecia, el café regular aumentó significativamente la glicemia en varones con sobrepeso. ${ }^{8}$ En 2014, en Japón, el café regular disminuyó significativamente la glicemia. ${ }^{9}$ En Perú, no se han encontrado estudios publicados al respecto.

El presente estudio se realizó con el objetivo de analizar el efecto del consumo de café regular y café descafeinado sobre la glicemia en adultos jóvenes.

\section{MATERIALES Y MÉTODO}

Se realizó un estudio experimental con grupo control pre y posprueba, triple ciego. La muestra fue de 42 estudiantes, 3 grupos de 14 personas cada uno; esta se calculó mediante el programa estadístico Minitab16 con los valores siguientes: 6 $\alpha=0,05$; desviación estándar asumida, 32; diferencia máxima, 40; potencia del objetivo, 0,80. La prueba de tolerancia oral a la glucosa (PTOG) fue la unidad de análisis. Se incluyeron estudiantes aparentemente sanos, índice de masa corporal (IMC) entre 18,5 y $30 \mathrm{~kg} / \mathrm{m}^{2}$, edad de 18 a 25 años; se excluyeron estudiantes con enfermedades metabólicas, cardiovasculares, gastrointestinales, función anormal hepática y/o renal, embarazo, antecedentes familiares de diabetes, hipertensión arterial u otras enfermedades cardiovasculares, consumo de medicamentos hipoglicemiantes e hiperglicemiantes, anticonceptivos orales, café mayor de $9 \mathrm{~g} / \mathrm{d}$, alcohol frecuentemente, hormonas y tabaco. Se consideraron las siguientes variables: consumo de café regular es la ingestión diaria de $6 \mathrm{~g}$ de café regular disueltos en $600 \mathrm{~mL}$ de agua; consumo de café descafeinado, ingestión diaria de $6 \mathrm{~g}$ de café descafeinado disueltos en $600 \mathrm{~mL}$ de agua y glicemia, concentración de glucosa en sangre medida por glucómetro Accu-Chek Active ${ }^{\circledR}$.

A 63 estudiantes voluntarios de la Facultad de Medicina de la Universidad Nacional de Trujillo (FMUNT) se les aplicó una ficha clínica, que incluyó: talla, peso, IMC, presión arterial, antecedentes personales y familiares y estilo de vida. Se seleccionaron a 42 que cumplieron con los criterios de inclusión y exclusión y que aceptaron participar en el estudio. Se distribuyeron aleatoriamente como sigue: grupo café regular (G1), grupo café descafeinado (G2) y grupo control (G3). Durante las 8 semanas siguientes; $\mathrm{G} 1$ consumió $6 \mathrm{~g} / \mathrm{d}$ de café regular, 2 g disueltos en $200 \mathrm{~mL}$ de agua después de cada comida (desayuno, almuerzo y cena); $G 2$, $6 \mathrm{~g} / \mathrm{d}$ de café descafeinado, $2 \mathrm{~g}$ disueltos en $200 \mathrm{~mL}$ de agua después de cada comida y $G 3$, un placebo a base de 5 gotas de colorante vegetal marrón disueltas en $200 \mathrm{~mL}$ de agua después de cada comida. El grupo investigador verificó diariamente que cada participante cumpliera con su régimen de consumo. Se evaluó la glicemia mediante la PTOG sugerida por Minsa, ${ }^{10}$ en tres momentos, antes del consumo de café, a las 4 semanas de iniciado el consumo y a las 8 semanas. Durante las 8 semanas de consumo de café, los participantes se comprometieron a conservar su actividad física y dieta habitual. Los participantes informaron su dieta durante los tres días previos a cada PTOG ${ }^{11}$ y se determinó su ingesta calórica usando la tabla peruana de composición de alimentos..$^{12} \mathrm{Se}$ controló la actividad física en la semana previa a cada PTOG, mediante el Cuestionario internacional de actividad física. ${ }^{13}$

Las consideraciones éticas aplicadas se desarrollaron según lo estipulado en la Declaración de Helsinki de octubre de 2013 para 
la investigación biomédica (no terapéutica) ${ }^{14}$ y el artículo 91 del Código de Ética del Colegio Médico del Perú. ${ }^{15}$ Cada participante dio su aprobación mediante la firma del consentimiento informado. Se contó con la aprobación del Comité Permanente de Investigación y el Comité de Ética de la FMUNT.

Se calcularon las medias de las glicemias de cada grupo: antes del consumo, a las 4 y 8 semanas y se evaluaron con la prueba $t$ de Student para muestras relacionadas pre y posprueba. Se aplicó ANOVA entre los grupos, a las que resultaron significativas se les aplicó prueba de Tukey. Se calcularon las varianzas de las variables edad, IMC, ingesta calórica total y actividad física y se aplicó la prueba de Levene. Todas la pruebas se consideraron significativas si $p<0,05$. Se usaron los programas Microsoft Excel 2010 y SPSS 20.

\section{RESULTADOS}

Las características basales de los tres grupos de la población estudiada -edad, IMC, ingesta calórica total y actividad física- fueron similares antes del consumo y permanecieron sin cambios significativos durante el estudio (Tabla 1). El promedio de edad para los grupos G1, G2 y control G3 fue de 21,86, 21,57 y 22,36 años, respectivamente.

En los resultados de la PTOG antes del consumo no hubo diferencias significativas entre las medias de los grupos (Tabla 2). A las 4 semanas, a los 0 minutos el G1 y el G2 muestran un aumento no significativo de $0,24 \%$ y $1,63 \%$, respectivamente, en relación a la glicemia antes del consumo y el G3, una disminución no significativa de 5,84\% y a los 120 minutos la glicemia del G1 muestra una disminución no significativa de $6,07 \%$ con respecto al valor antes del consumo y en los $\mathrm{G} 2$ y $\mathrm{G} 3$, un aumento no significativo de $4,05 \%$ y $4,98 \%$, respectivamente (Tabla 2); entre los grupos no se encontraron diferencias significativas a los 0 minutos ni a los 120 minutos de la PTOG (Tabla 2). A las 8 semanas, a los 0 minutos el G1 mostró una disminución no significativa de $3,76 \%$ en relación con la glicemia encontrada antes del consumo; el G2, un aumento no significativo de $4,04 \%$ y el G3, una disminución no significativa de $2,43 \%$ y a los 120 minutos existe una disminución significativa

Tabla 1. Prueba de homogeneidad de IMC (kg/m2), ingesta calórica total (Kcal), actividad física (MET)

\begin{tabular}{|c|c|c|c|c|c|c|c|c|}
\hline \multirow[t]{2}{*}{ Variables } & \multirow[t]{2}{*}{ Grupos } & \multicolumn{2}{|c|}{$\begin{array}{c}\text { Antes del } \\
\text { consumo (Inicio) }\end{array}$} & \multicolumn{2}{|c|}{4 semanas } & \multicolumn{2}{|c|}{8 semanas } & \multirow[t]{2}{*}{$\begin{array}{c}\text { Inicio vs } 4 \\
\text { sem vs } 8 \text { sem (p) }\end{array}$} \\
\hline & & Media & Varianza & Media & Varianza & Media & Varianza & \\
\hline & G1 & 24,37 & 11,99 & 23,90 & 13,58 & 24,18 & 11,74 & $0,99 *$ \\
\hline \multirow[t]{2}{*}{$\triangle I M C$} & $\mathrm{G} 2$ & 22,94 & 8,49 & 22,53 & 7,92 & 22,78 & 7,79 & $0,97^{\star}$ \\
\hline & G3 & 24,13 & 6,06 & 23,84 & 5,56 & 24,04 & 5,86 & $0,35^{*}$ \\
\hline \multicolumn{2}{|c|}{$\mathrm{G} 1$ vs $\mathrm{G} 2$ vs $\mathrm{G} 3$ (p) } & \multicolumn{2}{|c|}{$0,27^{\star}$} & \multicolumn{2}{|c|}{$0,26^{*}$} & \multicolumn{2}{|c|}{$0,27^{\star}$} & \\
\hline \multicolumn{9}{|l|}{ Ingesta } \\
\hline \multirow{2}{*}{$\begin{array}{l}\text { calórica } \\
\text { total }\end{array}$} & $\mathrm{G} 2$ & 2277,71 & 99285,45 & - & 65716,99 & 2275,43 & 87336,26 & $0,75^{\star}$ \\
\hline & G3 & 2319,93 & 181008,84 & - & 77316,75 & 2291,50 & 82736,73 & $0,07^{\star}$ \\
\hline \multicolumn{2}{|c|}{ G1 vs $G 2$ vs G3 (p) } & \multicolumn{2}{|c|}{$0,15^{\star}$} & \multicolumn{2}{|c|}{$0,80^{*}$} & \multicolumn{2}{|c|}{$0,93^{*}$} & \\
\hline & G1 & 2004,53 & 5377618,94 & - & 5377 618,94 & 2211,54 & 5289771,33 & $0,99^{*}$ \\
\hline \multirow{2}{*}{$\begin{array}{l}\text { Actividad } \\
\text { física }\end{array}$} & G2 & 2019,00 & 6290424,00 & - & 6290424,00 & 1938,86 & 6385464,59 & $0,95^{\star}$ \\
\hline & G3 & 1722,43 & 2773788,72 & - & 2697547,10 & 1725,64 & 2695588,86 & $0,72^{\star}$ \\
\hline \multicolumn{2}{|c|}{ G1 vs $G 2$ vs $G 3$ (p) } & \multicolumn{2}{|c|}{$0,44^{*}$} & \multicolumn{2}{|c|}{$0,38^{*}$} & \multicolumn{2}{|c|}{$0,42^{*}$} & \\
\hline
\end{tabular}




\section{Acta Méalica Pernana}

\begin{tabular}{|c|c|c|c|c|c|c|}
\hline & PTOG & $\begin{array}{l}\text { Momentos } \\
\text { de estudio }\end{array}$ & $\begin{array}{c}\text { G1 } \\
\text { Media }\end{array}$ & $\begin{array}{c}\text { G2 } \\
\text { Media }\end{array}$ & $\begin{array}{c}\text { G3 } \\
\text { Media }\end{array}$ & G1 vs $G 2$ vs $G 3$ (p) \\
\hline \multirow{6}{*}{$\boldsymbol{\Delta}$} & \multirow{6}{*}{0 minutos } & Antes del consumo & 91,07 & 91,86 & 94,14 & $0,700^{\star}$ \\
\hline & & 4 semanas & 91,29 & 93,36 & 88,64 & $0,444^{*}$ \\
\hline & & 8 semanas & 87,64 & 95,57 & 91,86 & $0,006^{*}$ \\
\hline & & $\begin{array}{l}\text { Antes del consumo } \\
\text { vs } 4 \text { semanas }(p)\end{array}$ & $0,952 \dagger$ & $0,458 \dagger$ & $0,136 \dagger$ & \\
\hline & & $\begin{array}{l}\text { Antes del consumo } \\
\text { vs } 8 \text { semanas }(p)\end{array}$ & $0,303 \dagger$ & $0,216 \dagger$ & $0,442 \dagger$ & \\
\hline & & $\begin{array}{l}4 \text { semanas vs } \\
8 \text { semanas }(p)\end{array}$ & $0,465 \dagger$ & $0,465 \dagger$ & $0,241 \dagger$ & \\
\hline \multirow{6}{*}{$\boldsymbol{\Delta}$} & \multirow{6}{*}{120 minutos } & Antes del consumo & 114,07 & 116,50 & 110,57 & $0,792^{*}$ \\
\hline & & 4 semanas & 107,14 & 121,21 & 116,07 & $0,057^{\star}$ \\
\hline & & 8 semanas & 98,00 & 123,28 & 113,21 & $0,000^{\star}$ \\
\hline & & $\begin{array}{l}\text { Antes del consumo } \\
\text { vs } 4 \text { semanas }(p)\end{array}$ & $0,283 \dagger$ & $0,472 \dagger$ & $0,533 \dagger$ & \\
\hline & & $\begin{array}{l}\text { Antes del consumo } \\
\text { vs } 8 \text { semanas }(p)\end{array}$ & $0,006 \dagger$ & $0,361 \dagger$ & $0,695 \dagger$ & \\
\hline & & 4 semanas vs 8 semanas $(p)$ & $0,039 \dagger$ & $0,679 \dagger$ & $0,563 \dagger$ & \\
\hline
\end{tabular}

de $14,09 \%$ en la glicemia del G1 con respecto a los resultados obtenidos antes del consumo y en los $\mathrm{G} 2$ y G3, un aumento no significativo de $5,82 \%$ y $2,39 \%$, respectivamente (Tabla 2 ); entre los grupos se encontraron diferencias significativas a los 0 minutos y a los 120 minutos de la PTOG (Tabla 2) y al aplicar la prueba de Tukey se encontraron diferencias significativas, a los 0 minutos entre $G 1$ y $G 2$ y a los 120 minutos entre G1 y G2 y entre G1 y G3 (Tabla 3).

\section{DISCUSIÓN}

Estudios refieren que el consumo de café puede estar asociado con variaciones de la glicemia. Algunos investigadores han reportado el efecto reductor del consumo de café regular sobre la glicemia, ${ }^{7,9}$ otros atribuyen este efecto al consumo de café descafeinado. ${ }^{5}$

El efecto reductor del consumo de café regular sobre la glicemia, observado en el estudio al

Tabla 3. Comparaciones múltiples de las glicemias $(\mathrm{g} / \mathrm{dL})$ entre grupos según momento de estudio

\begin{tabular}{|c|c|c|c|c|c|c|c|}
\hline $\begin{array}{l}\text { Momento } \\
\text { de estudio }\end{array}$ & PTOG & $\begin{array}{c}\text { G1 } \\
\text { Media }\end{array}$ & $\begin{array}{c}\text { G2 } \\
\text { Media }\end{array}$ & $\begin{array}{c}\text { G3 } \\
\text { Media }\end{array}$ & G1 vs G2 (p) & G1 vs G3 (p) & G2 vs G3 (p) \\
\hline$\triangle 4$ semanas & 120 minutos & 107,143 & 121,214 & 116,071 & $0,048^{*}$ & $0,277^{\star}$ & $0,646^{*}$ \\
\hline \multirow{2}{*}{$\Delta 8$ semanas } & 0 minutos & 87,643 & 95,571 & 91,857 & $0,004^{*}$ & $0,173^{*}$ & $0,252^{\star}$ \\
\hline & 120 minutos & 98,000 & 123,286 & 113,214 & $0,000^{*}$ & $0,029^{*}$ & $0,195^{*}$ \\
\hline
\end{tabular}


cabo de ocho semanas se podría explicar por su alto contenido de cafeína, esta tiene múltiples mecanismos que contribuyen a disminuir la glicemia, como activar la isoforma $\alpha 1$ del AMP proteína-cinasa, aumentar el número de transportadores de glucosa GLUT-4, estimular la secreción de insulina por las células $\beta .^{16,17}$ Otros componentes del café como ácido clorogénico, ácido cafeico y cofactor $\mathrm{Mg}^{2+}$, también influyen reduciendo la glicemia. ${ }^{18}$

Al cabo de ocho semanas el grupo que consumió café regular tuvo una reducción no significativa en la glicemia a los 0 minutos y una reducción significativa a los 120 minutos de la PTOG, en los grupos café descafeinado y control se observaron variaciones en la glicemia pero ninguna resultó ser significativa. Por lo tanto, el consumo de café regular disminuiría la intolerancia oral a la glucosa. La prevención de enfermedades como diabetes, dislipidemia e hipertensión arterial, comprende entre sus aspectos básicos un plan de alimentación adecuado, el café regular que no tiene un valor calórico intrínseco y que además disminuye la intolerancia a la glucosa sería ideal para formar parte de una dieta que prevenga la diabetes como lo refiere la Asociación Latinoamericana de Diabetes (ALAD) ${ }^{19}$

\section{CONCLUSIÓN}

El consumo de café regular disminuye significativamente la glicemia a los 120 minutos de la PTOG en adultos jóvenes, y el consumo de café descafeinado no tiene efectos significativos sobre la glicemia en adultos jóvenes.

\section{REFERENCIAS}

I. Gotteland M, De Pablo S. Café y Salud. Santiago: Instituto de Nutrición y Tecnología de los Alimentos (INTA), Universidad de Chile.2008.

2. Queirolo Bobadilla C. Promoción del consumo interno del café en el Perú: Lineamientos de estrategia. [Tesis de post-grado].Lima: Escuela de Post-grado. Pontificia Universidad Católica del Perú; 2010.

3. United States Department of Agriculture. Nutrient data for $142 \mid 4$, Coffee, instant, regular, powder [sedeWeb].USA:National Nutrient Database for
Standard Reference; 2010 [actualizada el 7 de junio del 2013; acceso 10 de junio de 2013]. Disponible en: http://ndb.nal.usda.gov/ndb/foods/show $14 \mid 85$ ? $f g=\&$ man $=\& \mid f a c e t=\&$ format $=\&$ count $=\& \max =25 \&$ offset $=\&$ sort $=\& q$ lookup=coffee.

4. European Food Information Council (EUFIC).Caffeine and Health [sede web].Bélgica: Dra. Josephine Wills, Directora general del EUFIC [actualizada el 5 de julio de 2013; acceso 7 de julio de 2013]. Disponible en: http://www.eufic.org/article/es/page/FTARCHIVE/artid/cafeina-salud/

5. Greenberg JA, Owen DR, Geliebter A. Decaffeinated coffee and glucose metabolism in young men. Diabetes Care. 2010;33(2):278-80.

6. Wedick NM, Brennan AM, Sun Q, Hu FB, Mantzoros CS, Van Dam RM et al. Effects of caffeinated and decaffeinated coffee on biological risk factors for type 2 diabetes: a randomized controlled trial. Nutr J. 20I I; 10(93): I 1 86-1475/289|-93.

7. Ohnaka K, lkeda M, Maki T, Okada T, Shimazoe T,Adachi M, et al. Effects of 16-Week consumption of caffeinated and decaffeinated instant coffee on glucose metabolism in a randomized controlled trial.J Nutr Metab. 2012; 20I2(9):I-9.

8. GavrieliAI, Fragopoulou E, Mantzoros CS,Yannakoulia M. Gender and body mass index modify the effect of increasing amounts of caffeinated coffee on postprandial glucose and insulin concentrations; a randomized, controlled, clinical trial. Metabolism. 2013;62(8):1099-106.

9. Pham NM, Nanri A, Kochi T, Kuwahara K, Tsuruoka H, Kurotani Kayo et al. Coffee and green tea consumption is associated with insulin resistance in Japanese adults. Metabolism.2014;63(3):400-8.

10. Agüero M,Arbañil H, Zavala R, Gonzáles V, Kusunoki L, Béjar R. Proyecto Guía técnica para el diagnóstico y manejo de la comorbilidad tuberculosis (tb) y diabetes mellitus ( $\mathrm{dm}$ ) en el primer nivel de atención. [Sede Web]. Perú: Ministerio de Salud; 2012 - [actualizada el 2 de julio de 2012; acceso 10 de junio de 2013]. Disponible en: http://www.parsalud.gob.pe/ phocadownload/fondomundial/ProdcutoMateriallEC/PAL/GUIA\%20DMTB.version\%20final.pdf.

II. Huamán-Saavedra J], Fogel-Silva BE, Escobar-Pairazamán PI, CastilloMinaya KY. Efectos de la ingesta de Plukenetia volubilis Linneo o "Sacha inchi" en el perfil lipídico de adultos jóvenes. Acta Med Per. 2012; 29(3): $155-60$.

12. Ministerio de Salud, Centro de Alimentación y Nutrición, Tablas peruanas de composición de alimentos 2008, Lima, Perú. Disponible en: http:// www.rvcta.org//magenes/TablasPeruanasDe ComposicionDeAlimentos. pdf.

13. Cuestionario internacional de actividad física.2002 Octubre. Disponible en: http://www.ipaq.ki.se/questionnaires/SpanIQSHL7SELFrev230802.pdf

14. Declaración de Helsinki de la AMM. Principios éticos para las investigaciones médicas en seres humanos.Asociación Médica Mundial.Disponible en: http://www.wma.net/es/30publications/ IOpolicies/b3/

15. Colegio Médico del Perú. Código de ética y deontología. [Monografía en internet]. Lima: Colegio Médico del Perú. [Consultado 14 de abril 2012] Disponible en: http://www.cmp.org.pe/doc norm/codigo etica cmp.pdf.

16. Egawa TI, Hamada T, Ma X, Karaike K, Kameda N, et al. Caffeine activates preferentially $\alpha \mathrm{I}$-isoform of 5'AMP-activated protein kinase in rat skeletal muscle.Acta Physiol (Oxf). 20I I; 201:227-38.

17. Bruton JD, Lemmens R, Shi CL, Persson-Sjogren S, Westerblad H,Ahmed $\mathrm{M}$, et al. Ryanodine receptors of pancreatic beta-cells mediate a distinct context-dependent signal for insulin secretion. FASEB J. 2003; I7(I):30I-3.

18. Rojo G, Morcillo S, Almaraz M, Soriguer F. Consumo de café y diabetes mellitus. Endocrinol Nutr. 2005;52(10):556-63.

19. Guías ALAD de diagnóstico, control y tratamiento de la Diabetes mellitus tipo 2. [sede Web]. Colombia:ALAD; 2012 - [actualizada el 23 de mayo del 2012; acceso I0 de junio de 2013]. Disponible en: http://web.archive. org/web/*/http://www.aladlatinoamerica.org/phocadownload/guias\%20 alad.pdf.

\section{Correspondencia}

Juan Jorge Huamán-Saavedra

jjhuamans@gmail.com

\section{Conflictos de interés}

El autor declara no tener conflictos de interés en el planteamiento, la ejecución de la investigación y la elaboración del artículo.

Fecha de recepción: 13 de noviembre de 2014

Fecha de aceptación: II de enero de 2015 